\title{
HISTORIA DEL HOSPITAL GENERAL O PROVINCIAL DE MADRID, CUNA DE UN SERVICIO DE UROLOGÍA CENTENARIO.
}

\author{
Ataulfo Sáiz Carrero.
}

Especialista Sénior de Urología (Uubilado). Hospital General Universitario Gregorio Marañón. Madrid. España.

\begin{abstract}
Resumen.- OBJETIVO: Dar a conocer el inicio del Hospital General de Madrid, sus ubicaciones, sus problemas económicos y sus personajes médicos, en especial su primer director médico, el doctor Pérez de Herrera. Así como las aportaciones científicas y de asistencia médica que proporcionó a los habitantes de Madrid y a los que venían de otras ciudades, buscando formación y asistencia.

MÉTODOS: Se han revisado escritos y libros de conocidos médicos, expertos en Historia de la Medicina y documentos sobre el Madrid antiguo y actual, y sus monumentos.
\end{abstract}

RESULTADOS Y CONCLUSIONES: La historia de este hospital, escuela y templo de la medicina, va unida a la historia de la Beneficencia Provincial de Madrid. Sus dificultades económicas fueron grandes para poder subsistir. En él, a finales del siglo XIX, se pudo consolidar uno de los más antiguos servicios de urología de España.
Palabras clave: Historia de la urología española. Hospital General de Madrid. Servicio de Urología.

Summary.- OBJECTIVES: To show the beginning of the General Hospital of Madrid, its localization, economical problems and medical protagonists, mainly the first medical director: Dr. Perez de Herrera. Also, to show its scientific and health-care contributions to the people of Madrid and other cities, coming in the search for training or health-care.

METHODS: We reviewed writings and books from wellknown doctors, experts on history of medicine, and documents about the old and new Madrid and its monuments.

RESULTS AND CONCLUSIONS: The history of this hospital, medical school and temple, is engaged with the history of Madrid's provincial Charity.

Economical difficulties were great to subside. At the end of the 19th century one of the eldest urology departments in Spain was consolidated.

Keywords: History of Spanish Urology. General Hospital of Madrid. Urology Service.

\section{INTRODUCCIÓN}

¿Cuál es aquella virtud, tan perfecta y sublimada, que aun el cielo es su morada, y de tanta beatitud que sin ella todo es nada? La Caridad

"Enigmas". C. Pérez de Herrera. (16) 
En 1499 los Reyes Católicos fundan el Hospital de Santiago de Compostela. Con ello inician una política hospitalaria que tenía como finalidad agrupar en un solo hospital los diferentes hospitalillos y enfermerías que existían en la ciudad, regidas por la Iglesia. En casi todos los edificios religiosos había centros de refugio para peregrinos, pobres y enfermos. Los monjes eran los encargados de curar, pero no podían realizar intervenciones quirúrgicas, pues el Concilio de Letrán, en 1139, se lo prohibió.

Después de este hospital compostelano fue creado otro en Granada, en 1504 y, poco después, los generales de Valencia y Zaragoza, así como el de La Latina en Madrid (fundado por Beatriz Galindo), que estaba situado en la calle Toledo esquina a la Plaza de la Cebada. En tiempos de Carlos I, se creó el Hospital de la Corte, más tarde llamado Hospital del Emperador y, a partir de la segunda mitad del siglo XVI, Hospital del Buen Suceso. En él ingresaba la servidumbre de la Casa Real y estaba situado, en la Puerta del Sol (5).

Estas nuevas instituciones, que ya tenían un carácter de servicio público, seguían teniendo un enfoque polifuncional. Decía Juan Luis Vives (14941540): "Doy el nombre de hospitales a aquellas instituciones donde los enfermos son mantenidos y curados; donde se sustentan un cierto número de necesitados; donde se educan a los niños y niñas; donde se crían los hijos de nadie; donde se encierran a los locos y donde los ciegos pasan la vida".

Este concepto no cambió hasta mediados del siglo XVIII, que es cuando se pensó que un hospital no era el lugar de los marginados sino el espacio de los enfermos $y$, por tanto un edificio exclusivo de la medicina.

A pesar de ello, aún en el siglo XIX, no todos cumplían este fin.

Felipe II asienta en Madrid la Corte en 1561 (Figura 1), y poco después realizó una política similar a la que iniciaron los Reyes Católicos, o sea, la de reagrupar los diferentes y múltiples establecimientos sanitarios. Había, sobre todo en Madrid, muchos hospitalillos, refugios y enfermerías que había que poner bajo la administración del gobierno. A esto se opuso la Iglesia, pues ello implicaba la desaparición de gran cantidad de cofradías y congregaciones. Tuvo que recurrir el Rey al Embajador que tenía en la Santa Sede, hombre de talento y de genio violento, Don Enrique de Guzmán, Conde de Olivares, padre del Conde-Duque. Gracias a su gran gestión se consiguió la autorización del Papa Pío V, para que se llevara a cabo dicha agrupación $(2,19)$.
Pero aún tuvieron que transcurrir 14 años, y fue en 1587 cuando se inició dicho agrupamiento, después de un Decreto dado por el Arzobispo de Toledo, don Gaspar de Quiroga. En este año se logró trasladar los hospitales menores con enfermos contagiosos e incurables al de Antón Martín (discípulo de San Juan de Dios) y los restantes al recién creado Hospital General (19).

Dicho Arzobispo de Toledo, por encargo del Rey contrató los servicios del médico Juan Bautista Neroni para que organizara las concentraciones hospitalarias en un hospital, que se llamó Hospital General de Santa Catalina. Se reunieron: el Hospital del Campo del Rey, que se encontraba cerca de la puerta de Segovia; el Hospital de Convalecientes, de la calle Ancha de San Bernardo; el Hospital de la Pasión, destinado a mujeres, que estaba situado en la zona cercana a la Plaza de la Cebada y que pronto volvió a este mismo lugar por falta de espacio; el Hospital del Amor de Dios, situado en la calle del mismo nombre, y el Hospital de San Ginés. Este último parece ser que tiene su origen en el hospital más antiguo de Madrid. Cuando aún la ciudad no era Corte, los cronistas hablan de un hospital que había en la zona del santuario de Nuestra Señora de Atocha, cuyo origen se remonta a la pequeña ciudad árabe, antes de ser conquistada por Alfonso VI. Carlos I trasladó dicho hospital a un lugar situado en la calle Arenal y tomó luego el nombre de Hospital de los Caballeros de San Ginés (5).

Les siguen en antigüedad el Hospital de San Lázaro y el Hospital de San Ricardo o de Héticos. El primero, también, fundado en tiempos mahometanos.

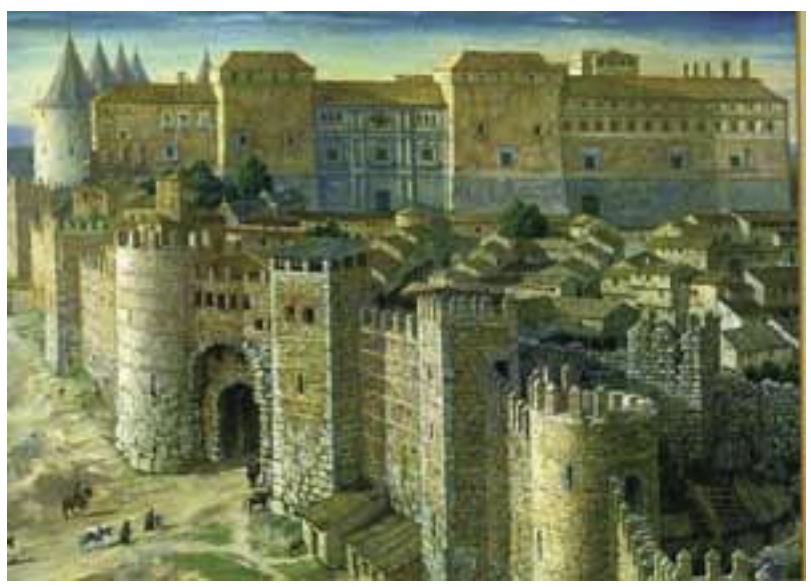

FIGURA 1. Madrid en 1561. Sus murallas y el Alcázar. 
En el segundo ingresaban los enfermos que padecían lepra, sarna o tiña. Desaparecieron con la fusión de los hospitales (19).

A pesar de la Orden Real, se siguieron creando pequeños hospitales, casas de misericordia y refugios, y alguien dijo en aquella época: "que pocas calles hay en esta Corte... en las que no haya iglesia, monasterio, parroquia u hospital".

El 8 de septiembre de 1596, después de una solemne procesión, se puso la primera piedra de un Albergue de Mendigos situado en un atochar de la zona sur de Madrid. El Rey Felipe II había permitido la construcción de dicho Albergue, que se gestionaría con limosnas y diferentes donaciones. La idea partió de uno de sus protomédicos: don Cristobal Pérez de Herrera (1556-1620), (Figura 2). Esta idea y muchas otras, las expuso en un libro publicado dos años después.

La primera institución para pobres y enfermos fue la fundada por San Juan de Dios en Granada: la Orden Hospitalaria (1550). Estas instituciones casi acapararon la mayoría de las organizaciones sanitarias y se extendieron por toda España. Pero en ellas, a diferencia de las que proponía Pérez de Herrera, sólo se buscaba mitigar la pobreza, pero no solucionarla.

\section{PÉREZ DE HERRERA Y SU ÉPOCA}

Este personaje, médico, político y poeta, había sido anteriormente Protomédico de las Galeras de España; se preocupó de atender a los pobres y mendigos que abundaban en la mayoría de las grandes ciudades y de diferenciar los verdaderos de los falsos.

Muy conocidos son los médicos renacentistas Vallés y Laguna, pero sin embargo, Cristobal Pérez de Herrera es muy poco conocido, aun por la clase médica, y poco valorado en general (la calle que lleva su nombre en Madrid, situada en el barrio de la Prosperidad, es insignificante, pues no llega a 40 metros de longitud) y sin embargo, se le debe considerar como uno de los hombres más interesantes de la medicina madrileña de aquella época.

Tal es así, que Lope de Vega le dedicó el siguiente soneto:

Que así acertase Herrera, ¿̇a quién no espanta? Cura por cierto ha sido peregrina, que dar al alma y cuerpo medicina, de la Física humana se adelanta.
Obra ha sido de Dios, que invidia tanta no sabe por los pasos que camina, grande el efeto, la intención divina, loable el modo, la paciencia santa.

Ni se podrá decir que ha errado Herrera el caminó evangélico que sigue:

heroica empresa que a la fama asombre.

O quién su nombre eternizar pudiera: pero la obra misma al tiempo obligue, que entrambos le darán inmortal nombre.

Era salmantino, de una familia de militares que había luchado en Europa con las tropas de los Reyes Católicos y de Carlos V.

Fue un personaje muy interesante. Durante su época de Protomédico de Galeras, 12 años, cuenta él mismo que llegó a conquistar siete banderas a turcos, ingleses y franceses. Durante este periodo de



FIGURA 2. El Dr. Cristóbal Pérez de Herrera (1556-1620). 
médico militar, un disparo de arcabuz le atravesó un hombro y estuvo muy grave, pero gracias a su gran fortaleza, sobrevivió. Protagonizó hechos heroicos y pintorescos, que han sido narrados primorosamente por Marañón (12).

Más tarde, dio un cambio radical a su vida. Su preocupación caritativa le surgió después de haber ejercido de médico en esos barcos. Las calamidades y desgracias que sufrían los condenados a galeras le cambiaron la actitud ante la vida (13).

Escribió cerca de 40 libros, algunos muy importantes. Destaca entre ellos uno que fue un resumen de los conocimientos médicos de aquella época: Compendivm totivs Medicinae ad tyrones, eis magna distinctione, \& claritate modum discendi \& provectoribus reminiscendi insinuans, in tres libros divisum... (Matriti 1614). En el libro tercero de este Compendio trata de la litiasis y de los trastornos de la micción (7).

Murió en la pobreza, pues no le reconocieron los servicios prestados a la Nación, además de sus gestas militares heroicas, el haber sido el principal fundador y primer director médico del Hospital General.

Siguió la línea iniciada por Luis Vives y continuada por Miguel Siginta, para acabar con el gran problema de los pobres, ordenar la Beneficencia y hospitalizar a los verdaderos enfermos.

En su libro, "Discurso de Amparo de pobres...", (Figura 3), Pérez de Herrera describe que había encontrado el sitio idóneo para edificar un albergue para los pobres. Era en el camino que iba a la iglesia de Nuestra Señora de Atocha, "azotada por vientos saludables y con un arroyo cercano de aguas puras". Consideraba, además, que había la posibilidad de trasladar el Hospital General a dicho Albergue, pues había sitio suficiente.

Opinaba en su libro " ...en el albergue de Madrid espero en Nuestro Señor se fabricará dentro dél, en la mitad del sitio, el Hospital General, que con por favor divino se ha de acomodar y trasladar dentro, por ser el sitio muy capaz desta gran fábrica".

Varias veces se refiere a lo mismo: "...y en otros dos patios a las espaldas desta casa, que confinan con estos, se podrá trasladar el Hospital General desta Corte porque al presente esta en sitio muy estrecho y poco airoso, y más metido en la Villa de lo que conviene para la salud della".

La primera aportación de dinero: $9.000 \mathrm{du}-$ cados, la recibió de la herencia del Cardenal don
Gaspar de Quiroga, pues de los 1.900 .000 ducados que dejó al morir, un tercio lo recibió el Papa, otro el Rey y el tercero lo dejó para sufragios y obras pías. Recibió, además, de la Junta de Policía, otros 7.000 ducados de la renta de sisa de Madrid. Como no fue suficiente esta cantidad para realizar su proyecto, pues necesitaba más de 50.000 ducados, tuvo que pedir limosna de puerta en puerta y hacer múltiples gestiones (4).

En sus escritos expone, claramente, el funcionamiento y la economía de estos albergues. Distingue en ellos un sitio para los enfermos y ancianos, donde serían atendidos debidamente, y otro para los pobres. Sobre estos últimos era necesario, en primer lugar, separar los simuladores, de los pobres verdaderos pues

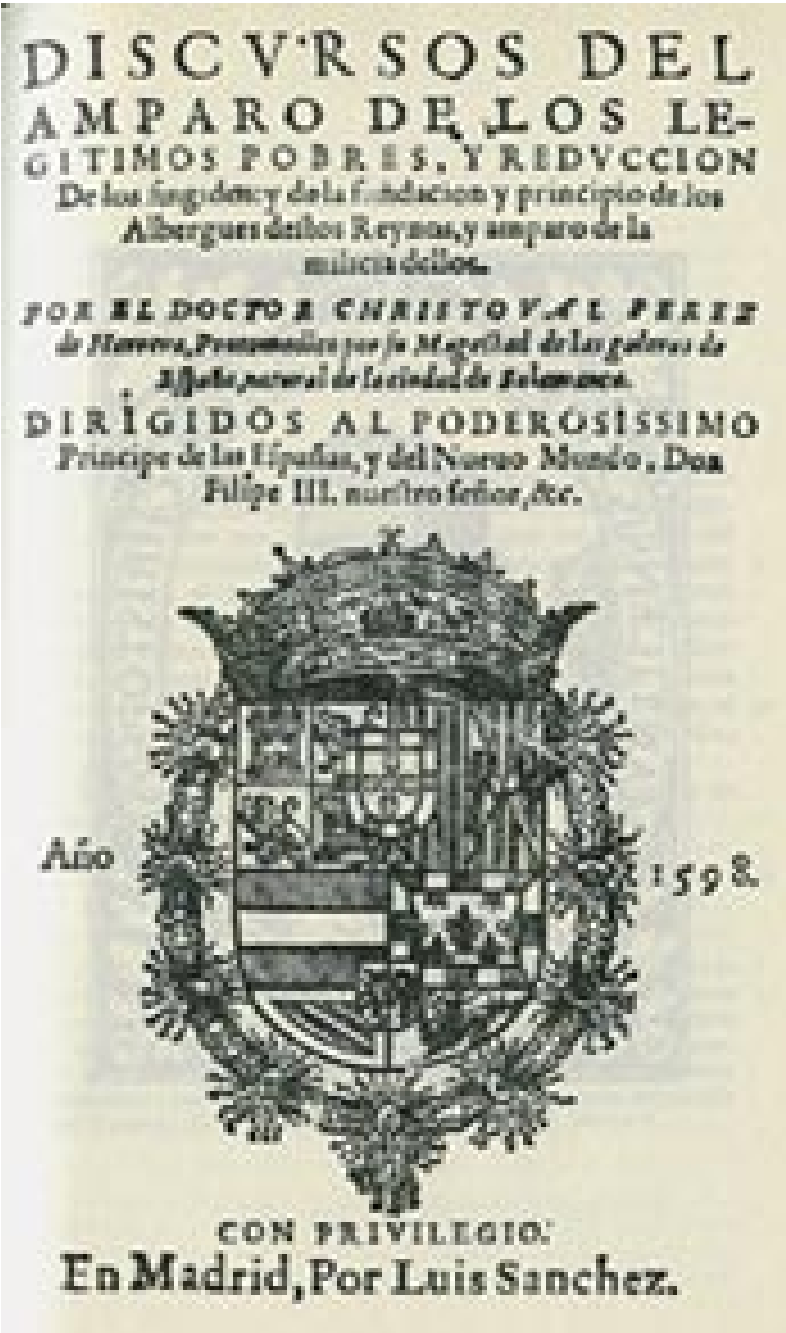

FIGURA 3. Libro del Dr. Pérez de Herrera publicado en 1598. 
hasta había espías de otros países extranjeros, que se camuflaban, disfrazados de menesterosos, y, lo que es peor, había quienes para mover a la caridad, y sacar más dinero, se producían autolesiones y hasta infligían heridas a sus propios hijos. A estos, hay que añadir los mutilados de las frecuentes guerras, la mayoría sin oficio ni beneficio (17).

Se preocupó también del amparo y formación de los niños abandonados o huérfanos; de la necesidad de obligar a los pobres que estaban sanos a trabajar en talleres; también dedica páginas a los pobres encarcelados; a los clérigos presos; a la redención de los cautivos; a los estudiantes pobres; así como "poner el remedio para corregir las vagabundas y delincuentes" (hubo un reformatorio de mujeres, en 1605-1606, con esta finalidad en un edificio anejo al Hospital General de Atocha: se llamaba "Galera Real").

Aunque el proyecto tenía enfoque nacional, se centró sobre todo en Madrid.

De la lectura del libro de Pérez de Herrera se desprende que la mayoría de los que mendigaban por las ciudades preferían hacer esto a trabajar.

Había tantos mendigos y pobres, que este autor recomendaba que no pasaran de una ciudad a otra y así poder tener un censo de ellos.

Además también quería solucionar el sentir de aquella sociedad que pensaba que "muchos de los mendigos vivían como gentiles, que no confesaban, ni iban a misa ni pensaban en la doctrina cristiana; que era gente sin cultura, sin razón, ni concierto; que eran peligrosos de contagiar enfermedades y además producían un espectáculo asqueroso y repugnante a las puertas de las iglesias y de otros lugares públicos".

La sociedad en general no dejaba de responder a esta demanda, pues iba muy bien con la moral social de la época, que consideraba que los que tenían posibilidades debían ayudar a los necesitados; pero eso no solucionaba el aumento creciente de menesterosos.

La sociedad del Renacimiento censuraba la ociosidad como enemiga del alma, pero al mismo tiempo considera el trabajo manual como una maldición. El trabajo era sinónimo de pobreza y vileza. Había pocas esperanzas de salir de apuros trabajando. Decía Mateo Alemán : "El dinero no se ganó a cavar". Esto fue muy negativo en la España renacentista, pues las actividades productivas estaban desprestigiadas (17).
Una manera muy frecuente de ganarse la vida era la de ser criado de un señor rico o noble, principalmente de paje, que le proporcionaba vestimenta elegante, educación y buenas maneras aprendidas de sus señores. Allí acudían principalmente los hijos de familias nobles con pocos recursos. Estos pajes o criados (los que se "crían" juntó un señor) no eran los únicos, pues alrededor de los grandes señores también había porteros, cocheros, mozos, cocineros, etc. Cuanto más importante era el señor más servidumbre tenía. Cuando iba a menos alguno de estos señores, se creaban gran cantidad de parados que preferían mendigar a tener trabajos duros y mal pagados (4).

Además, la oferta en las casas de los ricos y nobles no era suficiente para resolver los problemas del paro que planteaba el exceso de población en Madrid.

\section{UBICACIONES DEL HOSPITAL}

La primera reunión de todos los hospitales se realizó en 1587, en las Casas de Santa Catalina, situadas enfrente de donde actualmente está el edificio del Palacio de las Cortes, entre la calle del Prado y la Carrera de San Jerónimo. Se le llamó Hospital General de Nuestra Señora de la Encarnación y San Roque (Figura 4). Estas casas desaparecieron en tiempos de Mesonero Romanos (1803-1882) (1).

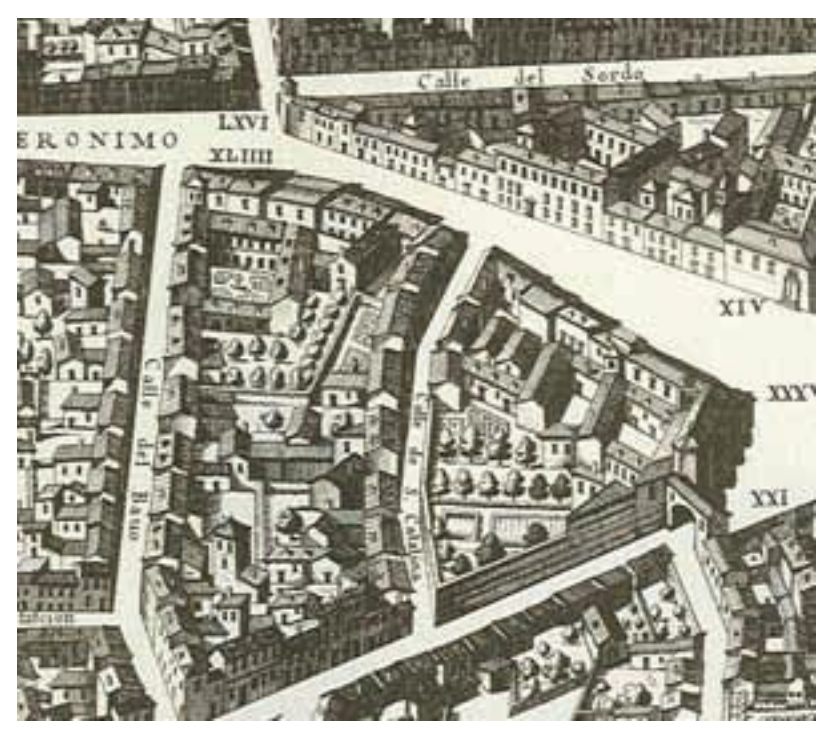

FIGURA 4. Zona de la primera ubicación del Hospital General. Plano de Texeira de 1656. 




FIGURA 5. El Hospital General en el plano de Texeira.

Poco tiempo después, se decidió su traslado, pues había poco espacio en este Hospital de Santa Catalina y además era un peligroso foco de infección, por estar en una zona céntrica de la ciudad (5).

Desde la perspectiva actual, no está claro si la amenaza era para los ciudadanos o para el Hospital, pues del Madrid de aquella época, en donde según Pérez de Herrera debían vivir "unas 300.000 almas", decía un cronista extranjero: "Tengo a esta Villa de Madrid por la más sucia y puerca de todas las de España, visto que no se ven por las calles otros que grandes servidores... que son grandes orinales de mierda, vaciados por las calles, lo cual engendra una fetidez inestimable y villana".

Se ordenó su traslado al Albergue de Mendigos, situado al final de la calle de Atocha (Figura 5). Con ello, se continuó con la tradición hospitalaria de aquella zona, que desde la época árabe se ha remontado hasta finales del siglo XX.

Pero hasta tiempos de Felipe III, no se realizó el traslado. El 9 de junio de 1603 se llevó el Hospital General de Hombres, pero el de la Pasión o de Mujeres fue trasladado en 1636. Pero no allí, sino a una zona cercana al anterior, en donde estaban situadas las casas de Don Juan Ruiz Gaytán de Ayala, en el lugar que luego estaría el Colegio de Cirugía de San Carlos.

Aproximadamente un siglo y medio después, Fernando VI desea construir un hospital nuevo. El primer proyecto se lo encargó a Ventura Rodríguez y lo rechazó por ser poco funcional. Sí aprobó el presentado por el ingeniero militar José Hermosilla. Este inicia en 1758 los trabajos del nuevo hospital en unos terrenos baldíos que había detrás del Albergue de

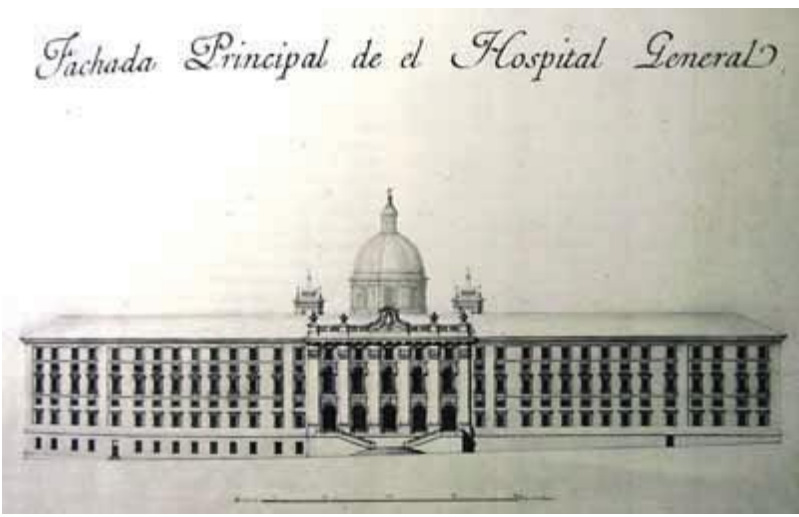

FIGURA 6. Proyecto de Sabatini. Fachada a la calle de Atocha.

Pérez de Herrera "para que mientras tanto siguiera el uso del antiguo". Construyó los sótanos y elevó el edificio en algunas zonas hasta el piso principal. A su muerte, ya en tiempos de Calos III, se lo encargan a Francisco Sabatini, quien lo reanuda con un tercer proyecto mucho más costoso y grandioso que el anterior. Incluía una solemne fachada situada en la calle de Atocha (Figura 6) (4).

Hubo grandes dificultades económicas para continuar con el monumental hospital, por lo que el proyecto fue abandonado. A la muerte de Carlos III, en 1788, solamente se había cerrado uno de los patios y se había construido un brazo que llegaba hasta la calle Atocha en la zona oeste (en donde está en la actualidad el Real Conservatorio de Música) (Figuras 7 y 8$)$.

En palabras de Sabatini, se construyeron sólo las dos quintas partes. Lo construido se inauguró en agosto de 1781, con 1561 camas (4).

En 1861 el Ayuntamiento compró por 1.380.711 pesetas la mayoría de su zona que daba a la calle de Atocha, y allí se construyeron casas de vecinos y una plaza (hoy de Sánchez Bustillo) (1).

Años después, en 1905, el proyecto de Hermosilla y Sabatini quedó reducido aún más, pues una parte de él hubo de ser cedida. Al no disponer la Diputación Provincial de casi recursos en aquella época, vendió al Estado el edificio que llegaba hasta la calle de Atocha, y en ellas se estableció el Hospital Clínico de la Facultad de Medicina.

La gran fachada de 165 metros que daría a esa calle fue sustituida por la actual, que es la cara de otro patio de los siete que se habían proyectados (4). 


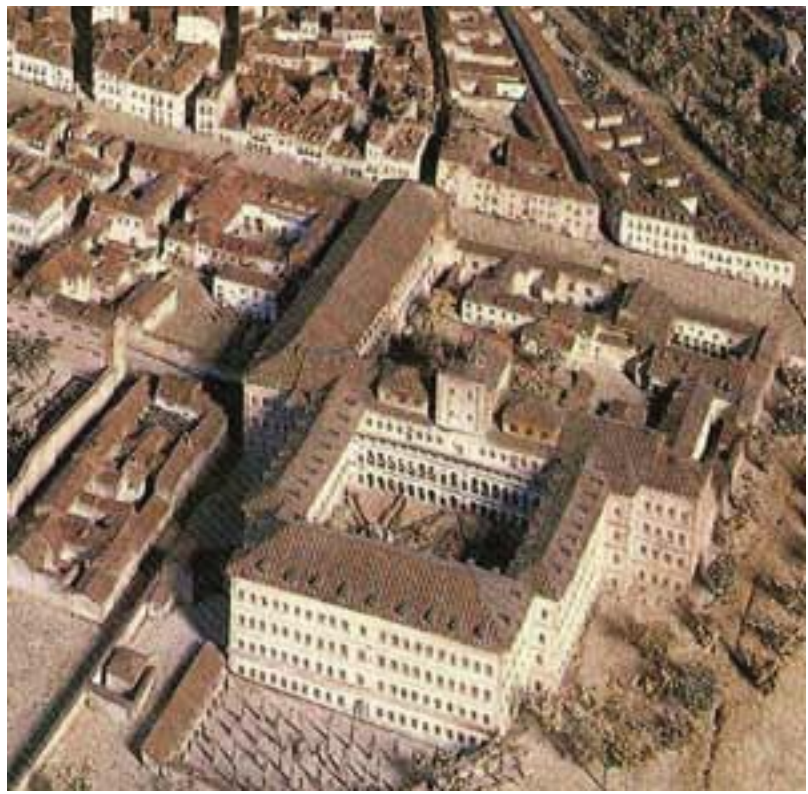

FIGURA 7. Maqueta de León Gil de Palacio de 1831 La calle Santa Isabel estaba cortada por el Hospital.

Posteriormente, en los años treinta del siglo $X X$, para aumentar el espacio e ingresar más enfermos, pues la demanda iba en aumento, se habilitaron las buhardillas en amplias salas. Se conservó el mismo orden arquitectónico del edificio y se consiguió aumentar un piso (Figura 9).



FIGURA 9. El antiguo edificio visto desde la Ronda de Atocha.

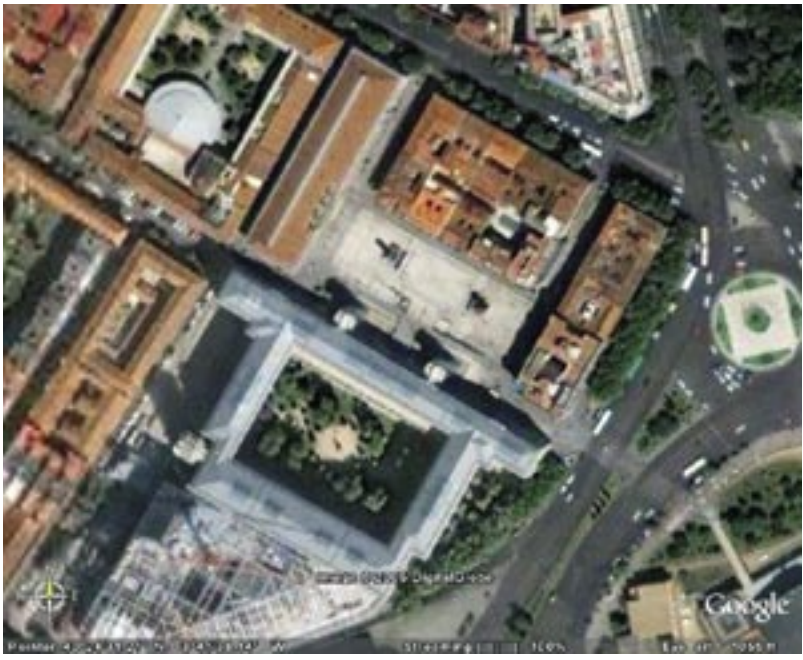

FIGURA 8. Sus edificios en la actualidad visto por vía satélite. La calle Santa Isabel se prolongó hasta la glorieta de Atocha en 1857.

Entonces el Hospital era un conjunto de grandes pasillos y amplias salas, destinadas unas a la estancia de mujeres y otras a la de los hombres. Había pocos espacios para métodos exploratorios, por lo que años más tarde, en 1968, sus dependencias fueron trasladadas a los edificios recién construidos en el lugar en donde estuvo situado el Hospital de San Juan de Dios, en la calle de Dr. Esquerdo, junto a los Hospitales Materno-Infantiles. A este conjunto arquitectónico se le denominó Ciudad Sanitaria Provincial Francisco Franco (Figura 10).

El edificio del antiguo Hospital logró salvarse a pesar de que había muchos que pedían su demolición. Gracias a un Real Decreto de 1977 fue declarado monumento histórico artístico (5).

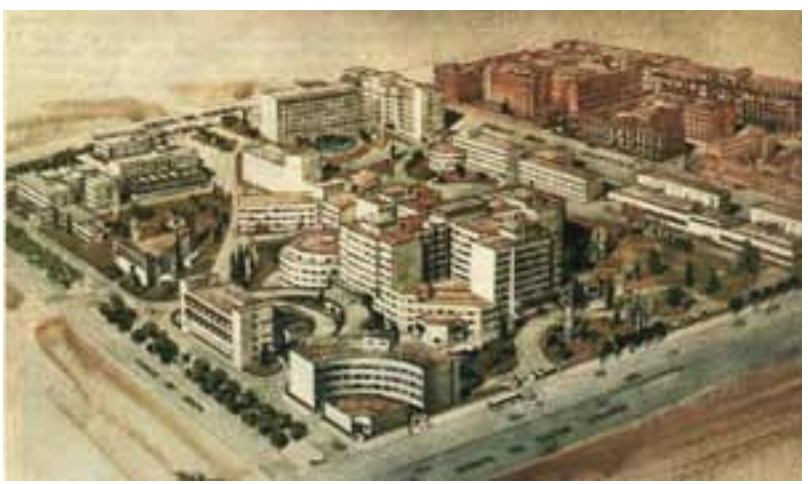

FIGURA 10. Ciudad Sanitaria Provincial Francisco Franco. 
Estuvo abandonado, lleno de escombros y de utensilios dejados u olvidados. Se llenó de gatos hambrientos y hasta se habló de la existencia de fantasmas que "sobrevivieron" en el edificio rehabilitado.

En el año 1980 comenzó su restauración con la finalidad de que fuera un museo. Fue realizada por Antonio Fernández Alba, y a finales del 1988 se llevan a cabo las últimas modificaciones, de entre las que cabría destacar especialmente las tres torres de ascensores, de vidrio y acero, diseñadas en colaboración con el arquitecto británico lan Ritchie (Figura 11).

El 10 de septiembre de 1992 Sus Majestades los Reyes inauguraron la Colección Permanente del Museo Nacional Centro de Arte Reina Sofía, que hasta este momento había acogido únicamente exposiciones de carácter temporal.

Últimamente ha sido ampliado este Museo con un edificio singular realizado por el arquitecto francés Jean Nouvel. Ha sido muy criticado tanto por su forma como por los materiales empleados (madera de la Amazonía, ilegal según Green Peace) (Figura 12).

\section{SU ADMINISTRACIÓN}

Felipe II, el 6 de diciembre de 1589, dotó al hospital de sus primeras Constituciones, redactadas por Bernardino de Obregón y en 1597 se le asignaron los beneficios de las ventas de la gramática de Nebrija.

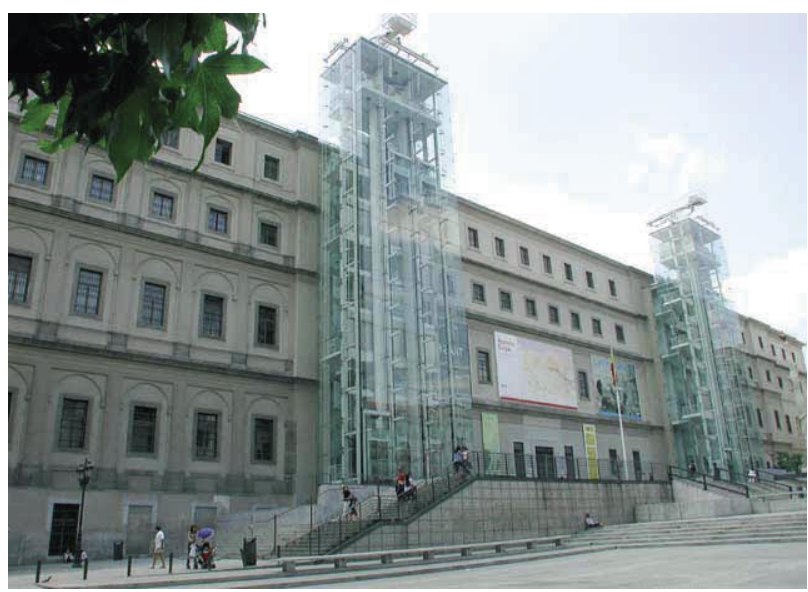

FIGURA 1 1. Fachada del Museo Reina Sofía.
Con motivo de recoger más fondos para el Hospital, Felipe II y el duque de Lerma, después de mucha rogativas, le concedieron una renta hecha sobre las sisas de la carne, que tampoco fue suficiente (1).

Una Real Cédula de 1613 ordenó que el Hospital tendría el privilegio para poder imprimir y vender el "arte de Antonio de Nebrija" en todos los dominios de España y se prohibió que lo hicieran otros (6).

Se le asignó una renta anual de 34.000 ducados, en tiempos de Felipe III (1616), que fue perpetuada por Felipe IV (1658). En el reinado de este monarca se le concedió, al cada vez más pobre Hospital General, una nueva sisa sobre la industria que era la más importante entonces en Madrid: la de las comidas. Esto se debió al Conde Duque de Olivares.

Pero en 1666 fue anulada, pues la miseria que se extendía por todo el país repercutió desgraciadamente en su economía.

Le llegó una nueva asignación en 1751, en tiempos de Fernando $\mathrm{VI}$, que además pagó con sus propios bienes las deudas del Hospital (más de

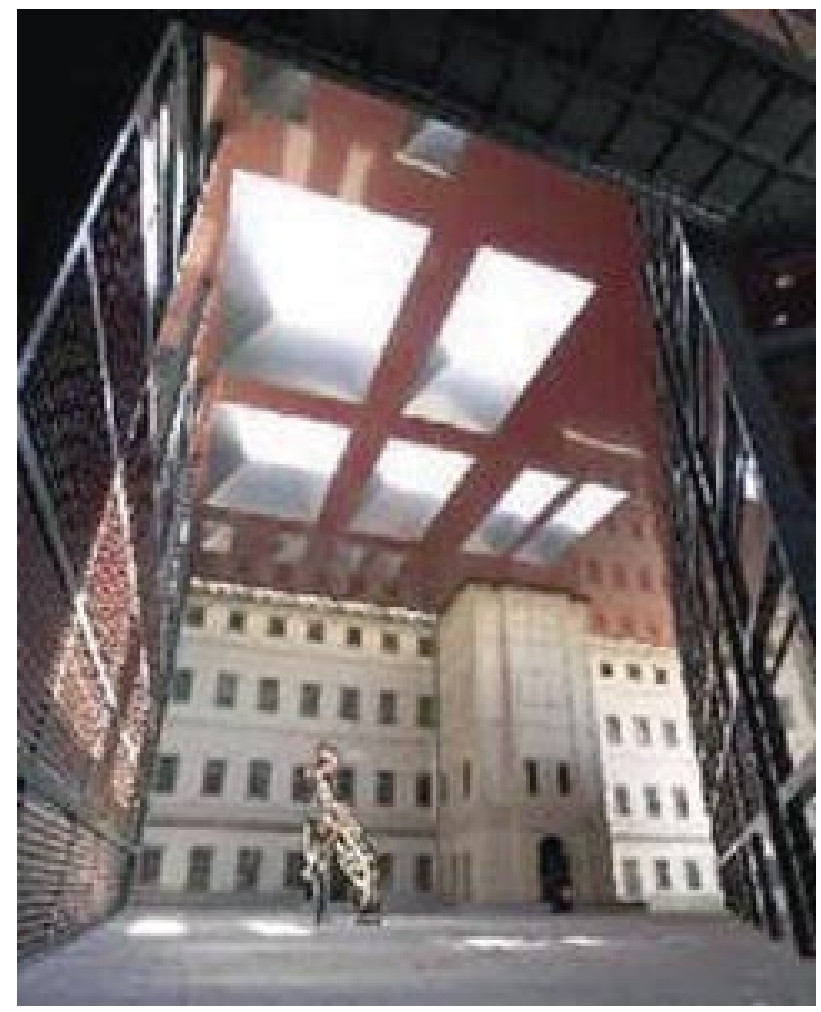

FIGURA 12. Ampliación del Museo Reina Sofía. 
1.200.000 escudos según Madoz), se le eximió de derechos reales y municipales, y se le adjudicaron las ganancias de la Plaza y de las Fiestas de las corridas de toros. "Lo que sirve de regocijo a los próceres que sirva de consuelo a los pobres" $(10,14)$.

Además, la administración del Teatro de los Caños del Peral (que representaba Ópera italiana), la fabricación y venta de alambre de hierro y la venta de El Almanaque del Gran Sarrabal de Milán. Los privilegios de la venta del libro de Nebrija seguían ayudando (1).

En 1760 Carlos III le concedió varios arbitrios y pensiones sobre mitras y promulgó otras Constituciones y nuevas Ordenanzas (6).

En 1849, fue regido y administrado por la Diputación Provincial; se le bautizó como Hospital Provincial de Madrid, unos veinte años más tarde (19).

\section{LABOR CIENTÍFICA Y ASISTENCIAL}

En la época de Felipe III, en 1603, se siguió con el intento de ordenar y centralizar la atención sanitaria madrileña.

Carlos II creó una plaza de Anatómico y en 1701, reinando Felipe $V$, se establece una Cátedra de Anatomía en el Hospital General, con la dotación de 3.600 reales anuales pagados por la Tesorería Real. Esta Cátedra tuvo gran prestigio y se explicaba en los sótanos del Hospital. A las clases no sólo acudían alumnos, sino muchos facultativos y público no médico. El primer profesor (1703) fue don José Arboleda, a quien sucedió, en 1707, Martín Martínez y en 1734 continuó Bernardo Araujo hasta 1762. Todos ellos eran médicos del hospital y tenían la obligación de explicar como mínimo doce lecciones al año sobre un cadáver (3).

A Araujo le sucedió Juan Gámez y, a éste, Antonio Dámaso de la Torrecilla, quinto y último de los catedráticos. Entonces existía una gran ignorancia sobre los conocimientos anatómicos, que se puede comprobar fácilmente mirando las contadas obras que se escribieron sobre este tema en aquella época. Los estudiantes acababan la carrera sin haber visto una sola disección (2).

El famoso Martín Martínez, que ya a los 22 años era Profesor por oposición en el Hospital General (14), comenta lo siguiente: "en nuestras universidades es sabido que no se hacen disecciones y si alguna se hace es burda y por puro cumplimiento; por lo que los maestros de anatomía son como catedráticos de anillo o profesores "in partibus", que sólo tienen el título pero no el ejercicio." Con ocasión de haber examinado a alguno de aquellos médicos para ascender a Protomédicos, al preguntarles sobre anatomía sacaba la conclusión que solo sabían que el hígado estaba a la derecha y el bazo a la izquierda.

Después de Felipe V, las Guerras de Sucesión llenaron el hospital de soldados heridos, pobres hambrientos y gente de la peor calaña, que eran mal alimentados y que dormían muchos de ellos en el suelo. Acabó siendo lo que en tiempos de Pérez de Herrera: un asilo y albergue de pobres (8).

Poco después de su coronación (1746), Fernando VI nombra director del Hospital a Juan Lorenzo del Real y le encarga que enseñe cirugía. Crea el primer Colegio de Cirugía de España, el Real Colegio de Cirujanos de San Fernando, a imitación de modelos franceses. Tuvo una vida corta, a consecuencia de la oposición del Protomedicato y de la Cofradía de San Cosme y San Damián Igremio de cirujanos y sangradores). Destacaron los cirujanos de la Corte: Tomás Duchernay, Andrés de Bereterrechea, Mateo Xiorro y Portillo y Juan de Diosa López. En su Estatuto se obligaba a realizar dos sesiones científicas al mes como mínimo (4).

En 1778 Antonio Gimbernat y Mariano Rivas, después de un viaje de estudios por Europa, son llamados para que se encarguen de organizar un colegio de cirugía en Madrid. A instancias del primero se creó el Real Colegio de Cirugía de San Carlos, mediante dos Reales Cédulas, concedidas en 1780 y 1787. En principio situado en los bajos del Hospital General, que ya se estaba construyendo bajo las órdenes de Sabatini. Las clases empezaron el 12 de octubre de 1787 y siguieron allí hasta 1830, año en el que se acabaron las obras de su nueva ubicación: el edificio construido en el solar del antiguo Hospital de La Pasión $(6,15)$.

Marañón aporta ideas deplorables del Hospital General durante el siglo XVIII. Pero según estudios posteriores, como los realizados por Âlvarez Sierra y Burke en 1977, el hospital durante el siglo $\mathrm{XVIII} \mathrm{no} \mathrm{era} \mathrm{peor} \mathrm{que} \mathrm{otros} \mathrm{europeos} \mathrm{de} \mathrm{esa} \mathrm{misma}$ época; su tasa de mortalidad no era muy elevada; su cuadro médico estaba constituido por profesionales bien pagados y prestigiosos y en sus salas se realizaba la medicina que se podía hacer en aquel momento. Aunque reconocen que los pacientes se apiñaban en pequeñas habitaciones carentes de ventilación y compartían varios la misma cama $(19,15)$. 


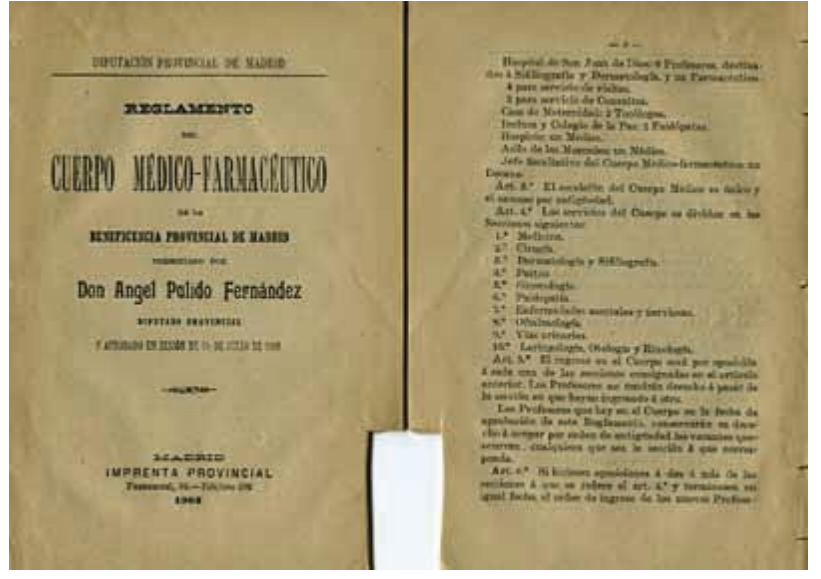

FIGURA 13. Reglamento del Hospital General de 1889. Creación de nuevas especialidades.

En 1795, siguiendo las ideas de Mariano Martínez Galinsoga se creó el Real Estudio de Medicina Práctica del Hospital General. Su finalidad era la de que algunos alumnos pudieran hacer sus dos años de prácticas obligatorias sin necesidad de recurrir a un médico privado, como era obligatorio hasta entonces. Fue dirigida está cátedra de medicina práctica por José Iberti y por José Severo López.

Después de la revolución de 1868 , se cerraron las Universidades y por tanto los Colegios de Medicina. Como no se formaban médicos, el Hospital creó una escuela libre de Medicina, la Escuela Teórico-Práctica de Medicina y Cirugía del Hospital Provincial de Madrid. Su director fue Martínez Leganés, acompañado de ilustres médicos como el Dr. Esquerdo (19).

Son dignos de mención los médicos del Hospital General de Madrid que, según Alvarez Salas, destacaron por sus estudios anatómicos:

Don Barto lomé Piñera y Siles, Don Juan Fernández Valle, Don Juan de Dios López, Don Tomás García Suelto, Don Cristóbal Pérez de Herrera, Don Celestino de Olózaga, Don Juan Blázquez, Don Manuel Santos Guerra, Don Juan de Navas, Don Juan Azoaga, Don Ramón Trujillo, Don Agustín Frutos, Don Antonio Dámaso Torrecilla, Don Mariano José de Larra, Don Juan Pablo Maroto, Don Pedro Custodio Gutiérrez, Doctor D. Agustín Recio, Doctor D. José Ventura Pastor, Doctor D. Francisco González, Doctor D. Pedro Aguilera, Doctor D. Serapio Escolar y Morales, Doctor D. José Rodríguez Villargoitia, Licenciado D. José Fernández, Don Rafael José de Guardia, Don Juan Luque y Luque, Don Bernardo Araújo y Ascárraga, Don José Arboleda, Don José Escolano (3).

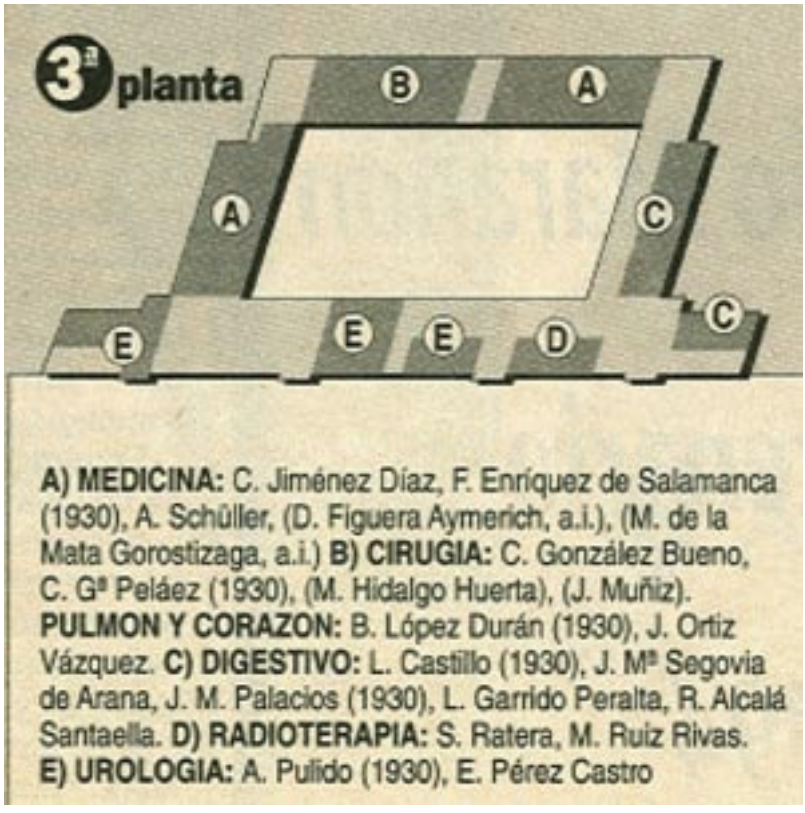

FIGURA 14. La planta tercera del Hospital desde 1930.

En este hospital hubo acontecimientos inéditos, como la introducción por primera vez en España de la anestesia etérea, la del cloroformo, el uso del termómetro clínico, el diagnostico con los rayos $X$, el suero antidiftérico, el Salvarsán, la primera sutura del corazón, y el primer cistoscopio en Madrid $(8,19)$.

El Dr. Pulido Fernández, siendo diputado provincial, presentó un nuevo Reglamento del Cuerpo Médico-Farmacéutico de la Beneficencia Provincial de Madrid, que fue aprobado en sesión el 15 de julio de 1889 (Figura 13). En él se creaban nuevas especialidades: Partos, Ginecología, Enfermedades mentales y nerviosas, Oftalmología, Vías urinarias y Otorrinolaringología $(1,18)$.

El Servicio de Vías Urinarias estaba situado en la tercera planta. En ella estaban, también, las salas de Cirugía, regidas por los doctores Antonio Gago, Hurtado y Ortiz de la Torre (Figura 14).

Su primer Jefe fue: D. Alfredo Rodríguez Viforcos hasta 1904, año en que murió prematuramente. Lo sustituyó el Dr. Antonio Bravo Piqueras, quien también murió pronto, en 1914. Le sucedió D. Isidro Sánchez-Covisa y Sánchez-Covisa, que ejerció la jefatura hasta 1939, que por razones políticas tuvo que dejarla. Se hizo cargo del Servicio D. Ángel Pulido Martín hasta que se jubiló en 1945. Por oposición tomó la jefatura D. Enrique Pérez Castro hasta su jubilación, ya en el nuevo edificio de la calle de Dr. Esquerdo $(9,11)$. 


\section{BIBLIOGRAFÍA y LECTURAS RECOMENDADAS ( ${ }^{*}$ lectura de interés $y^{* *}$ lectura fundamental)}

1. ALVAREZ SAINZ DE AJA, E.: "La Beneficencia Provincial de Madrid.(Datos de su historia 18611961)". Hosp. Gen. II:5, 1962.

**2. ALVAREZ SIERRA, J.: "Los hospitales de Madrid de ayer y de hoy". 201 páginas. Sección de Cultura e Información. Artes Gráficas Municipales. Tomo III. Madrid. 1952.

3. ALVAREZ SIERRA, J.: "Anatómicos madrileños famosos". 46 páginas. Instituto de Estudios Madrileños. Madrid. 1953.

*4. COMUNIDAD DE MADRID. VARIOS AUTORES.: "Francisco Sabatini 1721-1797". 347 páginas. Sociedad Editorial Electa España, S. A. Madrid. 1993.

5. DE SOROA Y PINEDA, A.: "El Hospital General de Madrid como escuela, templo, tradición y fortaleza señera de la Medicina de la Villa y Corte." Gaceta Médica Española. XXXVIII: 337, 1964.

6. GARCÍA BARRENO, P.: "El Hospital General de Madrid". En "Hospital General Universitario Gregorio Marañón”. Tomo I. Páginas 23-144. Espasa y Calpe, S.A. Madrid. 2001.

7. GRANJEL, L. S.: "El saber urológico en la Medicina Española del siglo XVII". Clín. Y Lab. LXXVI: 458, 1963.

*8. HIDALGO HUERTA, M.: "Evolución histórica del Hospital Provincial", Hosp. Gen., VIII: 22, 1968. (con este mismo título ha publicado un libro de 88 páginas en el año 2004, sin datos referenciales)

9. INSAUSTI CORDÓN, J. L.: "Compendio histórico de la Urología Española y de su Asociación". 258 páginas. Ponencia al IV Congreso Iberoamericano y XLVII Congreso Español de Urología. 1982.

10. MADOZ, P.: "Diccionario Geográfico-Estadístico-Histórico de España y sus posesiones de Ultramar". Facsímil de 1848. 623 páginas. Agualarga Editores, S.L. Madrid. 1999.
11. MAGANTO PAVÓN, E. \& AL. "Historia Biográfica y Bibliográfica de la Urología Española del siglo XX". Oficina de Historia de la Asociación Española de Urología. 384 páginas. Edicomplet. Madrid. 2000.

**12. MARAÑÓN, G.: "El pasado, el presente y el porvenir del Hospital General de Madrid".(Tomado de la Gaceta Médica Española, números 26 de abril, 2 y 9 de mayo de 1936, Madrid.) En: "Hospital General Universitario Gregorio Marañón". Tomo I. Páginas 5-22. Espasa Calpe S.A. Madrid. 2001.

13. MARAÑ́́N, G.: "La vida en las galeras en tiempo de Felipe II". En Vida e Historia. Páginas 94123. Octava edición. Espasa-Calpe, S.A. Madrid. 1962.

14. MUÑOZ CALERO, A.: "El Hospital General en la vida médica madrileña." Hosp. Gen. I: 8, 1961.

*15. NUÑEZ OLARTE, J.M.: "El Hospital General de Madrid en el siglo XVIII". 356 páginas. Cuadernos Galileo de Historia de la Ciencia. N $^{\circ} 19$. C.S.I.C. Madrid. 1999.

16. PÉREZ DE HERRERA, C.: "Enigmas". 158 páginas. Ediciones Atlas. Madrid. 1943.

**17. PÉREZ DE HERRERA, C.: "Discursos Del Amparo De Los Legítimos Pobres, Y Reducción De los fingidos: y de la fundación y principio de los Albergues destos Reynos, y amparo de la milicia dellos". 1598. 316 páginas . En "Cristobal Pérez de Herrera. Amparo de pobres". Clásicos castellanos. Edición, introducción y notas de Michel Cavillac. Espasa Calpe, S.A. Madrid. 1975.

18. REGLAMENTO DEL CUERPO MÉDICO-FARMACÉUTICO DE LA BENEFICENCIA PROVINCIAL DE MADRID. Presentado por Don Ángel Pulido Fernández. 52 páginas. Imprenta Provincial. Madrid. 1903.

**19. VALLADARES ROLDÁN, R.: "El Hospital Provincial de Madrid". En: "Hospital General Universitario Gregorio Marañón”. Tomo II. 157 páginas. Espasa Calpe S.A. Madrid. 2001. 